\title{
SPATIAL PATTERNS IN HOUSEHOLD DEMAND
}

\author{
By AnNe C. CASE ${ }^{1}$
}

\begin{abstract}
In this paper I discuss economic processes that may give rise to spatial patterns in data, and explore the relative merits of alternative modeling approaches when data are spatially correlated. Specifically, I present an estimation scheme that allows for spatial random effects, and focus attention on cases in which such a framework may be preferred to the more general fixed effects framework that nests it. I use the models presented, together with information on the location of households in an Indonesian socio-economic survey, to test spatial relationships in Indonesian demand for rice.
\end{abstract}

Keywords: Spatial correlation, Moran I statistics, random effects models, fixed effects models, SUSENAS.

\section{INTRODUCTION}

MANY ECONOMIC PROCESSES have spatial aspects. Unobservable variables may be spatially correlated and thereby produce spatial correlation in the errors of equations describing economic behavior. Examples are climate, soil quality, or the availability of substitute goods that are not recorded in data sets. In addition, if households directly gain utility in consuming bundles similar to those consumed by their neighbors, one may observe spatial correlation in household behavior. ${ }^{2}$

In many cases, fixed effect models can be used to control for spatial components. This can be done, for example, by incorporating either household level effects into a demand equation, using panel data, or village level effects, using a cross-sectional demand survey. ${ }^{3}$ This strategy for controlling spatial effects is often a suitable one.

There are, however, some cases in which a spatial modeling framework may be more appropriate. There may be no intraregional variation in a variable of interest, for example when price depends upon distance from some point. When this is true, the responsiveness of demand to changes in such variables cannot be examined in a fixed effect framework. The variables of interest are perfectly correlated with the fixed effects. In other cases, there may be specific interest in the spatial components themselves. For example, in testing the extent to which households look to a reference group when making decisions, the magnitude and direction of interactions between households may be of primary importance. This information would be difficult to extract from a fixed effect framework. Finally, if a spatial process is responsible for regional effects, a spatial

\footnotetext{
${ }^{1}$ I thank David Card, Angus Deaton, Mark Gersovitz, Ann Harrison, Larry Katz, Daniel Sichel, and anonymous referees for comments on an earlier draft.

${ }^{2}$ In Linear Expenditure Systems developed by Gaertner (1974) and Pollak (1976), and in an Almost Ideal Demand System estimated by Alessie and Kapteyn (1985), the behavior of other households affects a given household's behavior through social proximity. Such a metric may also be used with the model presented below. Future work might also incorporate a dynamic process through which learning occurs.

${ }^{3}$ For example, Deaton (1987) models household demand with a cluster specific fixed effect.
} 
model that constrains random effects to be spatially correlated is more efficient than a fixed effect model that does not.

This paper examines spatial models that may be preferred to fixed effect models when spatial parameters are parameters of interest or when fixed effect modeling is infeasible or inefficient. Section 2 discusses tests for the presence of spatial patterns in economic data, and economic processes that may induce such patterns. In addition, it presents an estimation scheme that allows for spatial interaction among households. Section 3 nests the spatial models in a more general fixed effect framework, and discusses cases in which one framework may be preferred to the other. Section 4 presents an example of spatial modeling. Information on the location of households in an Indonesian socio-economic survey is used to explore spatial relationships in Indonesian demand for rice.

\section{SPATIAL MODELS}

Spatial patterns can often be described in terms of spatial correlation, the positive or negative correlation of a variate between neighboring regions of a surface. One can test for spatial correlation on any surface that has been divided into nonoverlapping contiguous districts. In Figure 2.1, a map of Bali Province in Indonesia, districts sharing a boundary are said to share a join. In this figure, a first order spatial process can be characterized by the relationship between the value a variate $Y$ takes in one district and the value it takes in joining districts. For example, a first order relationship between district 2 and its neighbors, districts 1,8 , and 3 , can be described:

$$
Y_{2}=\phi Y_{1}+\phi Y_{8}+\phi Y_{3}+\varepsilon_{2}
$$

where $\varepsilon$ is a vector of identically, independently distributed errors, and subscripts refer to districts. ${ }^{4}$

Spatial correlation in a variate may not, by itself, be of much interest. For example, if wealthier households tend to live in the western districts of Bali Province, then there would be little information in finding that demand patterns in the western districts resemble each other. Instead, it would be more useful to test for spatial correlation in the residuals of a regression that removed wealth effects, such as equation (2.1):

$$
Y=X \beta+\varepsilon .
$$

The simplest test for spatial correlation is the Moran I (MI) statistic (Moran (1950)), which can be used with (2.1) to measure covariance in errors between joining districts relative to the variance in errors in a given district. Assigning unit weight to all joins ( $\omega_{i j}=1$ if districts $i$ and $j$ share a join) and zero weight

\footnotetext{
${ }^{4}$ See Cliff and Ord (1981), Griffith (1988), or Anselin (1988) for an introduction to stochastic spatial processes.
} 


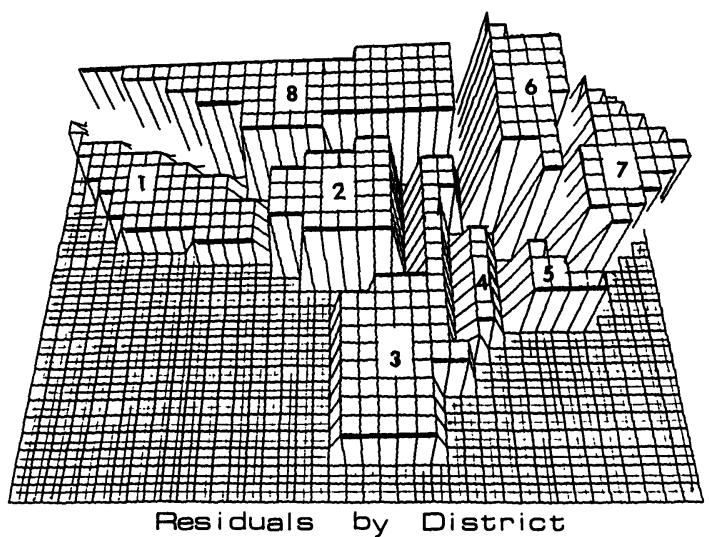

Figure 2.1

Weights used in the analysis of spatial correlation between Indonesian districts, Bali province

BALI DISTRICTS

\begin{tabular}{|c|c|c|c|c|c|c|c|c|}
\hline & $d=1$ & $d=2$ & $d=3$ & $d=4$ & $d=5$ & $d=6$ & $d=7$ & $d=8$ \\
\hline$d=1$ & 0 & .5 & 0 & 0 & 0 & 0 & 0 & .5 \\
\hline$d=2$ & .33 & 0 & .33 & 0 & 0 & 0 & 0 & .33 \\
\hline$d=3$ & 0 & .25 & 0 & .25 & 0 & .25 & 0 & .25 \\
\hline$d=4$ & 0 & 0 & .33 & 0 & .33 & .33 & 0 & 0 \\
\hline$d=5$ & 0 & 0 & 0 & .33 & 0 & .33 & .33 & 0 \\
\hline$d=6$ & 0 & 0 & .2 & .2 & .2 & 0 & .2 & .2 \\
\hline$d=7$ & 0 & 0 & 0 & 0 & .5 & .5 & 0 & 0 \\
\hline$d=8$ & .25 & .25 & .25 & 0 & 0 & .25 & 0 & 0 \\
\hline
\end{tabular}

elsewhere, the $M I$ statistic for the residuals of model (2.1) is

$$
M I=\frac{\sum_{i} \sum_{j} \bar{e}_{i} \omega_{i j} \bar{e}_{j} / 2 J}{\sum_{i} \bar{e}_{i}^{2} / d}
$$

where $\bar{e}_{i}$ is the average of residuals for observations in district $i, J$ is the total number of joins, and $d$ is the total number of districts.

Under the null hypothesis that errors are identically, independently distributed normal variates, the Pitman-Koopmans theorem can be used to evaluate the moments of the $M I$ statistic. Under fairly weak assumptions, the $M I$ statistic is asymptotically normally distributed. (See Appendix One.)

A significant $M I$ statistic is consistent with several spatially regressive structures. Many of these appear as special cases of the following model:

$$
\begin{aligned}
& Y=\phi W Y+X \beta+u=(I-\phi W)^{-1} X \beta+(I-\phi W)^{-1} u, \\
& u=\tau W u+\varphi+\varepsilon=(I-\tau W)^{-1} \varphi+(I-\tau W)^{-1} \varepsilon .
\end{aligned}
$$

For $T$ households in $N$ districts, $W$ is a $(T N \times T N)$ weighting matrix that assigns to households in district $i$ the average value of variate $Y$ in districts 
surrounding district $i{ }^{5}$ An example of such a weighting matrix is given in Figure 2.1 for Bali Province for the case of one observation per district. In this example, all neighbors of a district are given equal weight, and all districts are equally influenced by their neighbors taken together $\left[\Sigma_{j} w_{i j}=1\right]$. These assumptions may be altered if more information about the relative importance of neighbors is available. The coefficient $\phi$ measures the extent to which households are emulating their neighbors, gaining utility by consuming bundles similar to neighbors' bundles (WY).

In (2.2), the error term $u$ has three components. $\varepsilon$ is a $(T N \times 1)$ vector of random errors, with $E(\varepsilon)=\iota_{T N} 0, \operatorname{Var}(\varepsilon)=\sigma_{\varepsilon}^{2} I$, and $E\left(X^{\prime} \varepsilon\right)=\iota_{k} 0$. $\varphi$ is a nonspatial district specific error. For household $k$ in district $i, E\left(\varphi_{k}\right)=0 ; E\left(\varphi_{k} \varphi_{j}\right)$ $=\sigma_{\varphi}^{2}$ if $j \in i$; else $E\left(\varphi_{k} \varphi_{j}\right)=0$. In this section, it will be assumed that $E\left(X^{\prime} \varphi\right)$ $=\iota_{k} 0$. Potential correlation between explanatory variables $X$ and the district specific error component $\varphi$ will be discussed in Section 3. In addition to $\varphi$ and $\varepsilon$, the error in equation (2.2) contains a spatial error term ( $\tau W u)$, with the interpretation that the error terms for observations in any district $i$ contain $\tau$ times the average error found in districts surrounding district $i(W u)$. Spatial correlation in errors $(\tau \neq 0)$ may result when unobserved spatially correlated variables drive demand. Any unobserved regional differences, such as unobserved differences in the availability of substitute goods or in regional economic conditions, may result in unobserved errors being different in different parts of a country, but related in nearby areas.

In addition, for neighboring districts $i$ and $j$, I assume that district $i$ 's right side variables are uncorrelated with $j$ 's errors. For this reason, district $i$ 's conditioning variables affect $j$ 's consumption only through their effect on $i$ 's consumption. Correlation between $i$ 's conditioning variables and consumption in $j$, net of the effect of $j$ 's own conditioning variables, identifies $\phi$. After controlling for the direct effect of neighbors' consumption, the residual correlation between consumption in districts $i$ and $j$ identifies $\tau$.

For a model with error components $(\tau W u+\varphi)$, given $\phi=0$, ordinary least squares estimation is inefficient. If OLS is performed without error correction, the parameter estimates $\beta$ are still unbiased, but the variance estimates are biased and may lead to faulty inference. Furthermore, standard random effects modeling that ignores the spatial component of the error is also inefficient.

The presence of the term $\phi W Y$ in model (2.2), representing the influence neighbors have on demand through their behavior, has important effects both on the estimation and the interpretation of the coefficients. If the ordinary least squares estimator $\hat{\beta}=\left(X^{\prime} X\right)^{-1} X^{\prime} Y$ is used when the true model is (2.2), and $\phi$ is not equal to zero, the parameter estimates of $\beta$ will be biased and inconsistent, even when $\tau=0$.

\footnotetext{
${ }^{5}(I-\phi W)$ is invertible for all $\{\phi \mid-1<\phi<1\}$. One can prove this by demonstrating $|I-\phi W|$ $=\Pi_{j}\left(1-\phi e_{j}\right)$, where $e_{j}$ is the $j$ th eigenvalue of $W$, and proving that $-1<e_{j} \leqslant 1$ for all eigenvalues, $e_{j}$.
} 
In addition, the presence of these interaction terms alters the interpretation of the $\beta$ coefficients. In the absence of interaction terms between districts, the effect on the dependent variable of a change in the $k$ th explanatory variable is $\beta_{k}$. However, with interaction between districts, the effect of a change in the $k$ th explanatory variable is $C^{i i} \beta_{k}$, where $C^{i i}$ is the $(i, i)$ element of $(I-\phi W)^{-1}$. A change in an exogenous variable in district $i$ affects demand in district $i$, which also affects demand in district $i$ 's neighbors. Demand in district $i$ changes both because of the change in the explanatory variable, and also because of change in its neighbors' demand. These interaction effects are captured in $(I-\phi W)^{-1}$, and vary between districts. In a restricted way, then, the model allows the effect of a change in an exogenous variable on the dependent variable to be district specific.

Because ordinary or generalized least squares estimation of (2.2) leads to biased and inconsistent parameter estimates if $\phi$ is not equal to zero, maximum likelihood estimation is used. The log likelihood function for (2.2) is

$$
L=\frac{-T N}{2}[\ln (2 \pi)]+\ln |A|+\ln |C|-\frac{N}{2} \ln |V|-\frac{1}{2} \sum_{i=1}^{N} G_{i}^{\prime} V^{-1} G_{i}
$$

where

$$
G_{i}=Y_{i}-(\phi+\tau) W_{i} Y+(\phi \tau) W_{i}^{2} Y-X_{i} \beta+\tau W_{i} X \beta .
$$

$Y_{i}$ and $X_{i}$ are $(T \times 1)$ and $(T \times k)$ observations from district $i . W_{i}$ are the $(T \times T N)$ rows of matrix $W$ that correspond to observations in district $i ; W_{i}^{2}$ are the analogous rows of $W^{2} ;[\ln |A|+\ln |C|]$ is that part of the Jacobian of the transformation from errors into $Y$ due to spatial components, with $A=$ $(I-\tau W), C=(I-\phi W)$; and for each district the inverse of the variancecovariance matrix of $(\varepsilon+\varphi)$ is

$$
V^{-1}=\frac{1}{\sigma_{\varepsilon}^{2}}\left[I_{T}-\frac{\sigma_{\varphi}^{2}}{\sigma_{\varepsilon}^{2}+T \sigma_{\varphi}^{2}} \iota \iota^{\prime}\right] .
$$

The asymptotic variance-covariance matrix for $\left[\sigma_{\varepsilon}^{2}, \sigma_{\varphi}^{2}, \beta, \phi, \tau\right]$ is given by the inverse of the information matrix.

I use an iterative procedure to obtain the maximum likelihood estimates. I obtain estimates of $\beta, \phi$, and $\tau$ from a spatial model that constrains nonspatial district effects to be zero $(\varphi=0)$. In this case, $V=\sigma_{\varepsilon}^{2} I$, and the likelihood can be concentrated with respect to $\beta$ and maximized with respect to $\phi$ and $\tau$. From these estimates of $(\beta, \phi, \tau)$ and the first order conditions of likelihood (2.3), I obtain estimates of $\sigma_{\varepsilon}^{2}$ and $\sigma_{\varphi}^{2}$ :

$$
\begin{aligned}
& \hat{\sigma}_{\varepsilon}^{2}=\frac{1}{N(T-1)} \sum_{i=1}^{N} \hat{G}_{i}^{\prime}\left(I_{T}-\frac{1}{T} \iota \iota^{\prime}\right) \hat{G}_{i}, \\
& \hat{\sigma}_{\varphi}^{2}=\frac{1}{N} \sum_{i=1}^{N} \bar{G}_{i}^{2}-\frac{1}{T} \hat{\sigma}_{\varepsilon}^{2},
\end{aligned}
$$


where $\hat{G}_{i}$ is $G_{i}$ evaluated at the updated estimates for $(\beta, \phi, \tau)$, and $\bar{G}_{i}$ is the mean of $\hat{G}_{i}$. I use these estimates of $\hat{\sigma}_{\varepsilon}^{2}$ and $\hat{\sigma}_{\varphi}^{2}$ to find the values of $\beta, \phi$, and $\tau$ that maximize (2.3). I then use these estimates to update $\sigma_{\varepsilon}^{2}$ and $\sigma_{\varphi}^{2}$, and repeat this process until convergence.

Under the assumption that the parameters $(\beta, \phi, \tau)$ are independent from those in $V, v=\left(\sigma_{\varepsilon}^{2}, \sigma_{\varphi}^{2}\right)$, and that the values of $v$ that maximize the likelihood are such that: (i) $\operatorname{det} V \geqslant \lambda>0$; (ii) $V$ is nonnegative definite; and (iii) $v^{\prime} v \leqslant M$ for arbitrary positive numbers $\lambda$ and $M$, the iterative procedure converges to a solution of the first order maximizing conditions of the likelihood function. This is proven through simple extension of Oberhofer and Kmenta (1974).

In deriving large sample approximations for the estimators, the number of districts is held constant. Instead the sample size increases by increasing the number of observations per district. As a result, estimates obtained for $\beta, \tau, \phi$, and $\sigma_{\varepsilon}^{2}$ are consistent and asymptotically efficient.

One can test for the presence of spatial correlation in errors and a spatially correlated dependent variable by using Wald, Lagrange multiplier, or likelihood ratio tests of the hypothesis that $\phi$ or $\tau$ equals zero.

The likelihood and the parameter estimates from spatial models can also be compared with those from a fixed effect model that does not constrain the effects. Such a fixed effect model is discussed in the next section.

\section{A NESTING FIXED EFFECT MODEL}

Spatial models embedded in (2.2) are random effects models, where part of the effects are restricted to be correlated in space and the error remaining, $[\varphi+\varepsilon]$, is assumed to be uncorrelated with the right-hand-side variables. Model (2.2) can be rewritten:

$$
Y=X \beta+\varepsilon+[\phi W Y+\tau W u+\varphi]=X \beta+\varepsilon+f
$$

where the term in brackets is identical for every observation within a district. $f$ is a $(T N \times 1)$ vector of constrained random effects. For observation $k$ in district $i,(2.2)$ restricts $f$ :

$$
f_{k}=\left[\phi W_{k} Y+\tau W_{k} u+\varphi_{i}\right]
$$

where $W_{k}$ is the $k$ th row of weighting matrix $W$. That is, model (2.2) assigns to the random effect of each observation within a district: $\phi$ times the average value of $Y$ in districts surrounding the observation's district plus $\tau$ times the average error in districts surrounding the observation's district plus a nonspatial district specific error $\varphi_{i}$.

For this reason, the $\beta$ parameters of (2.2) can be estimated using a least squares dummy variable estimator of the corresponding fixed effect model:

$$
Y=X \beta+D \theta+\varepsilon
$$

where $D$ is a $(T N \times(d-1))$ matrix of dummy variables.

There are cases in which fixed effect modeling may be preferred to spatial modeling. Fixed effect models relax a key assumption of the spatial models. In 
its assumption that conditioning variables are uncorrelated with error $\varphi$, the spatial model (2.2) is a special case of a more general model that does not maintain this assumption. In those cases in which $E\left(X^{\prime} \varphi\right) \neq \iota_{k} 0$, the parameter estimates from the spatial models will be inconsistent. A Hausman test of the difference between the estimates from the spatial model and those from a fixed effect model tests for the presence of such correlation. The fixed effect model is inefficient in the absence of correlation between conditioning variables and district specific errors, but provides consistent estimates in their presence.

While there are cases in which fixed effect models may prove to be superior, there are also cases in which subsuming spatial effects within fixed effects results in a real loss in information. For example, a research goal may be the determination of appropriate reference groups in demand. The appropriateness of different weighting matrices for neighbor assignment, $W$, can be tested in the spatial framework above by nesting different weighting matrices, say $W^{1}$ and $W^{2}$ :

$$
W=\alpha W^{1}+(1-\alpha) W^{2}
$$

and varying $\alpha$ between 0 and 1 to maximize the likelihood (2.3). While these neighbor assignments may be based on geography, as in the example above, they need not be. In addition, one may test whether households mimic their demographic neighbors, their neighbors in income space, or some combination of the above. For example, if reference group is thought to depend upon income, distance between any two districts $i$ and $j$ could be measured: $\omega_{i j}=1 /$ income $i$-income $j \mid$, for $i \neq j$, normalized so that $\sum_{j} \omega_{i j}=1$. The information of who is influenced by whom may be harder to extract from fixed effects. While choice of metric to measure distance in any given variable is arbitrary, in research based on U.S. states using other states as reference points, Case, Hines, and Rosen (1989) found their answers were insensitive to the metric chosen.

In addition, spatial models distinguish the extent to which influence from the reference group is registered. A value of $\phi$ near zero suggests neighbors have little influence, while a value of $\phi$ near 1 or -1 suggests that households are strongly influenced by neighbors' purchases. The value of $\phi$ may vary between different goods, suggesting that for some purchases households are sensitive to consumption in a reference group, but for other purchases they are not. Moreover, one might be interested in testing whether neighbors' influence is stronger within certain income groups, or in a particular age range. Estimates of $\phi$ are provided by spatial models, but not by fixed effect models. Subsuming $\phi$ in the fixed effects in those cases where it is a parameter of interest results in an information loss.

Furthermore, for policy purposes one may wish to control behavior through manipulation of exogenous variables. In doing so, if households gain utility in consuming bundles similar to their neighbors' $[\phi \neq 0]$, one will want to account for expected interhousehold effects $[\phi W Y]$ when modeling anticipated reaction to change in an exogenous variable. Fixed effect models such as (3.3), which do not allow one to judge whether an observed spatial pattern is due to correlation 
in the dependent variable or correlation in errors, do not allow such effects. When interhousehold effects are important, the ultimate effect of a change in an explanatory variable could be misinterpreted in the fixed effect framework provided by (3.3).

Finally, spatial models may provide estimates in cases where one would like to control for regional effects using a fixed effect framework but, because of data constraints, one cannot. For example, if data lack intraregional variance, fixed effects are perfectly correlated with variables of interest. Because of the constraints placed on the regional effects by the spatial models, one can obtain estimates of parameters of interest in a framework that controls for regional effects, albeit in a constrained way.

An example of spatial modeling, and a comparison of spatial and fixed effect estimates, are presented in the final section.

\section{DEMAND FOR RICE IN INDONESIA}

As an example of spatial modeling, this section will examine demand for rice purchased in Indonesian markets. The Indonesian socio-economic survey SUSENAS draws samples from every district in the country. Districts, roughly comparable in size to counties in the eastern U.S., are the contiguous units upon which neighborliness is measured. I use data on 141 districts in the 11 provinces for which maps were available. Districts were selected for analysis if they (1) imputed positive rent values for homeowners; (2) consumed rice purchased in markets rather than that grown at home; and (3) were adjacent to at least one other district in the analysis. $(T N=2089$.) There are on average 2 villages surveyed in each district. Prices used here are village mean log unit values. Tests for bias induced by using unit values in lieu of prices found no significant bias in parameter estimates.

In the demand equation estimated, from which residuals are tested for spatial correlation, the log quantity of market rice purchased by a household $(Y)$ is modeled as a function of the log household expenditure per household member ( $\ln X P C$ ), the size of the household (MEMS), the number of household members above the age of 10 (GT10), and the mean village log prices of market rice $\left(P_{\text {rice }}\right)$, fish $\left(P_{\text {fish }}\right)$, housing $\left(P_{\text {hous }}\right)$, and fuel $\left(P_{\text {fuel }}\right)$ :

$$
\begin{aligned}
Y= & \beta_{0}+\beta_{1} \ln X P C+\beta_{2} M E M S+\beta_{3} G T 10 \\
& +\beta_{4} P_{\text {rice }}+\beta_{5} P_{\text {fish }}+\beta_{6} P_{\text {hous }}+\beta_{7} P_{\text {fuel }}+\varepsilon .
\end{aligned}
$$

It is assumed initially that $\varepsilon$ are normally distributed errors, $E(\varepsilon)=\iota 0$, and $\operatorname{Var}(\varepsilon)=\sigma^{2} I$. The results of the regression appear in column 1 of Table IV.1. The expenditure elasticity $(0.13)$ is equal to the income elasticity for rice estimate for Indonesia by Barker and Herdt (1980) using a demand equation and data similar to those used here. Ceteris paribus, an additional household member is associated on average with an increase in rice purchases of roughly $18 \%$. An additional adult in the household, holding constant household size and per capita expenditure, is associated on average with an increase in rice 
TABLE IV.1

Maximum Likelihood Estimates of Models with Spatial Components

Model 4.1 No district specific effects or spatial correlation $(\varphi=\tau=\phi=0)$.

Model 4.2 District specific effects, but no spatial correlation $(\varphi \neq 0, \tau=\phi=0)$.

Model 4.3 Spatial correlation in dependent variable $(\varphi \neq 0, \tau=0, \phi \neq 0)$.

Model 4.4 Spatial correlation in errors $(\varphi \neq 0, \tau \neq 0, \phi=0)$.

Model 4.5 Spatial correlation in both $(\varphi \neq 0, \tau \neq 0, \phi \neq 0)$.

\begin{tabular}{|c|c|c|c|c|c|}
\hline \multirow[b]{2}{*}{$\begin{array}{l}\text { Explanatory variables }{ }^{\mathrm{a}} \\
\text { (standard errors) }\end{array}$} & \multicolumn{5}{|c|}{ Model Estimates } \\
\hline & $(4.1)$ & $(4.2)$ & $(4.3)$ & $(4.4)$ & $(4.5)$ \\
\hline Log expend per household member & $\begin{array}{l}0.1259 \\
(.0173)\end{array}$ & $\begin{array}{l}0.1111 \\
(.0154)\end{array}$ & $\begin{array}{l}0.1101 \\
(.0154)\end{array}$ & $\begin{array}{l}0.1094 \\
(.0158)\end{array}$ & $\begin{array}{l}0.1095 \\
(.0154)\end{array}$ \\
\hline Number of household members & $\begin{array}{l}0.1762 \\
(.0064)\end{array}$ & $\begin{array}{l}0.1679 \\
(.0060)\end{array}$ & $\begin{array}{l}0.1670 \\
(.0060)\end{array}$ & $\begin{array}{l}0.1667 \\
(.0056)\end{array}$ & $\begin{array}{l}0.1667 \\
(.0060)\end{array}$ \\
\hline Number of adults in household & $\begin{array}{l}0.0195 \\
(.0081)\end{array}$ & $\begin{array}{l}0.0314 \\
(.0080)\end{array}$ & $\begin{array}{l}0.0323 \\
(.0078)\end{array}$ & $\begin{array}{l}0.0329 \\
(.0071)\end{array}$ & $\begin{array}{l}0.0329 \\
(.0078)\end{array}$ \\
\hline Village log price market rice & $\begin{array}{r}-0.4786 \\
(.0830)\end{array}$ & $\begin{array}{r}-0.3978 \\
(.1049)\end{array}$ & $\begin{array}{r}-0.4607 \\
(.1042)\end{array}$ & $\begin{array}{r}-0.4190 \\
(.1073)\end{array}$ & $\begin{array}{r}-0.4210 \\
(.1064)\end{array}$ \\
\hline Village log price fish & $\begin{array}{l}0.0018 \\
(.0256)\end{array}$ & $\begin{array}{l}0.0343 \\
(.0296)\end{array}$ & $\begin{array}{l}0.0293 \\
(.0292)\end{array}$ & $\begin{array}{l}0.0313 \\
(.0295)\end{array}$ & $\begin{array}{l}0.0314 \\
(.0295)\end{array}$ \\
\hline Village log price fuel & $\begin{array}{l}0.2631 \\
(.0334)\end{array}$ & $\begin{array}{l}0.0605 \\
(.0369)\end{array}$ & $\begin{array}{l}0.0512 \\
(.0372)\end{array}$ & $\begin{array}{l}0.0477 \\
(.0352)\end{array}$ & $\begin{array}{l}0.0479 \\
(.0374)\end{array}$ \\
\hline Village log price housing & $\begin{array}{l}0.0295 \\
(.0095)\end{array}$ & $\begin{array}{r}-0.0251 \\
(.0126)\end{array}$ & $\begin{array}{r}-0.0323 \\
(.0124)\end{array}$ & $\begin{array}{r}-0.0341 \\
(.0123)\end{array}$ & $\begin{array}{r}-0.0339 \\
(.0128)\end{array}$ \\
\hline $\begin{array}{r}\tau \text {-coefficient of spatial } \\
\text { correlation in errors }\end{array}$ & - & - & - & $\begin{array}{l}0.4529 \\
(.0804)\end{array}$ & $\begin{array}{l}0.4401 \\
(.0956)\end{array}$ \\
\hline $\begin{array}{l}\phi-\text { coefficient of spatial } \\
\text { correlation in dep var }\end{array}$ & - & - & $\begin{array}{l}0.3970 \\
(.0737)\end{array}$ & - & $\begin{array}{l}0.0152 \\
(.0420)\end{array}$ \\
\hline$\sigma_{\varepsilon}^{2}$-household variance & 0.1124 & 0.1088 & 0.1088 & 0.1088 & 0.1088 \\
\hline$\sigma_{\varphi}^{2}-$ district variance & - & 0.0958 & 0.0687 & 0.0646 & 0.0649 \\
\hline Chi-square test statistic ${ }^{b}$ & $\begin{array}{r}1161.26 \\
(0.99)\end{array}$ & $\begin{array}{l}41.58 \\
(0.99)\end{array}$ & $\begin{array}{l}5.70 \\
(0.91)\end{array}$ & $\begin{array}{c}0.03 \\
(0.09)\end{array}$ & \\
\hline
\end{tabular}

\footnotetext{
${ }^{a}$ Intercept not reported. Standard errors are estimated using the outer product of first partial derivatives of the log likelihood function.

${ }^{\mathrm{b}}$ LR test of equality in log likelihood between each column and column 5. Probability of correctly rejecting null hypothesis of equality in likelihoods is presented in parentheses.
}

consumption of $2 \%$, suggesting that adults consume only slightly more rice than children do. The own-price elasticity of market rice is -0.48 .

The MI statistic for model (4.1), without any spatial structure, equals 0.4676 , and is significantly different from its expected value $(-0.0076)$. The $t$ statistic $(T=22.24)$ is significant on a $99.5 \%$ confidence interval. That neighboring districts have related residuals can also be seen in Figure 2.1, which maps mean residuals by district for Bali Province. It is apparent that northern districts have the higher mean residuals, and southern districts, the lower ones. Taken together, these indicators suggest that modeling a spatial component to demand is appropriate.

The results of estimating the spatial random effects model (2.2) and fixed effect model (3.3) are presented in Tables IV.1 and IV.2. In Table IV.1, the parameter estimates of an OLS model with no spatial components are compared to the spatial model's estimates. Column 2 presents results of relaxing the 
TABLE IV.2

A Comparison of Estimates from Random EfFects AND FiXed EFFECTS MOdel

\begin{tabular}{lcc}
\hline \hline & $\begin{array}{c}\text { Model } 2.2 \\
\text { Spatial correlation } \\
\text { in errors } \\
(\tau \neq 0)\end{array}$ & Model 3.3 \\
$\begin{array}{l}\text { Explanatory variables } \\
\text { (standard errors) }\end{array}$ & 0.1094 & 0.1095 \\
\hline Log expend per household member & $(0.0158)$ & $(0.0159)$ \\
& 0.1667 & 0.1668 \\
Number of household members & $(0.0056)$ & $(0.0057)$ \\
Number of adults in household & 0.0329 & 0.0327 \\
& $(0.0071)$ & $(0.0071)$ \\
Village log price market rice & -0.4190 & -0.4078 \\
& $(0.1073)$ & $(0.1143)$ \\
Village log price fish & 0.0313 & 0.0326 \\
& $(0.0295)$ & $(0.0309)$ \\
Village log price fuel & 0.0477 & 0.0304 \\
& $(0.0352)$ & $(0.0359)$ \\
Village log price housing & -0.0341 & -0.0382 \\
& $(0.0123)$ & $(0.0134)$ \\
\hline
\end{tabular}

Source: SUSENAS May 1978. Number of observations $=2089$; number of districts $=141$.

Hausman test of equality in coefficient estimates from (2.2) and (3.3) $=0.32$. Probability of correctly rejecting null of equality $<0.005$.

assumption of no nonspatial district specific effect $(\varphi)$. The increase in likelihood over OLS is roughly 500 points. This immediately suggests that the OLS standard errors reported in column 1 are biased. When the preferred specification of Model 4.4 below $\left[\sigma_{\varepsilon}^{2}=0.1088, \sigma_{\varphi}^{2}=0.0646, \tau=0.45\right]$ is used to correctly calculate the OLS standard errors, they are one third to one half again as large as those presented in column 1 .

Columns 3 and 4 allow for spatial correlation in the dependent variable $(\phi \neq 0)$ and in errors $(\tau \neq 0)$ respectively. Both models achieve an increase in likelihood over column 2 of roughly 20 points, and a large reduction in the district specific variance $\sigma_{\varphi}^{2}$, which falls from 0.09 to 0.06 as part of the district specific error is attributed to a spatial component. Results from both models can be used to reject a null hypothesis that the overall district level random effects are identically, independently distributed. Model 4.3 finds a large, significant spatial component $(\phi=0.40$, std. error $=0.07)$ in the dependent variable, while Model 4.4 finds a large, significant spatial component $(\tau=0.45$, std. error $=0.08)$ in the errors. In order to distinguish between these models, they are nested in Model 4.5.

Figure 4.1 provides a contour map of log likelihood values for a range of $\tau$ and $\phi$ for Model 4.5. This map suggests that the two parameters of spatial correlation substitute for each other empirically. There is a distinct ridge along which the highest likelihoods obtain. This ridge occurs where higher values of $\tau$ are matched with lower values of $\phi$, and vice versa. While there are two local maxima, roughly at $\tau=0$ and at $\phi=0$, the higher of the two is clearly at ( $\phi=0$, 


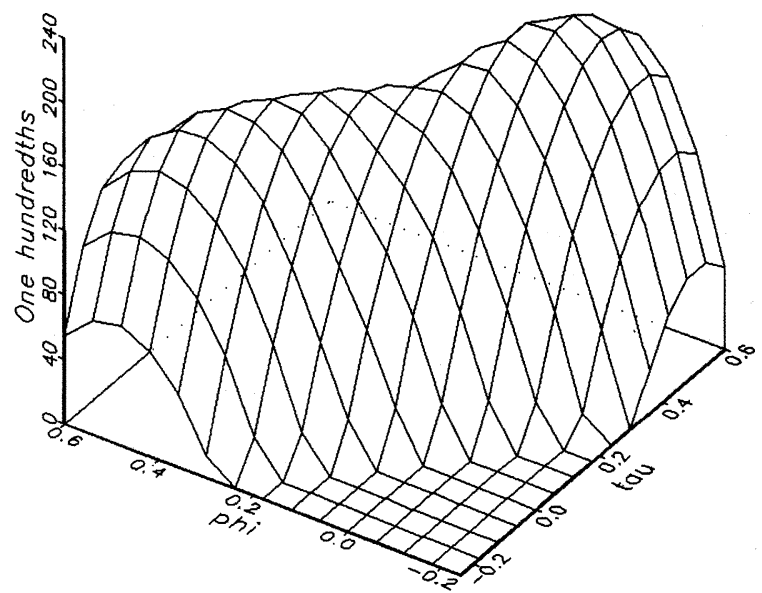

FIGURE 4.1.-Log likelihood contours.

$\tau=0.45)$. Without nesting the models with spatial correlation in the dependent variable $(\phi \neq 0, \tau=0)$ and in the errors $(\tau \neq 0, \phi=0)$ in a broader framework, it would not be possible to determine whether spatial correlation should indeed be attributed to the errors or to the dependent variable. If one brings to the estimation no theoretical prior as to which process underlies the observation of spatial correlation, the two potential processes must be nested as in Model 4.5 in order to learn about their relative importance.

The results of Table IV.1 suggest that spatial models, in addition to providing information on the underlying spatial process, also increase the efficiency of the estimation. However, the efficiency and information gains are more apparent than real if the district specific effects are correlated with right side variables. For this reason, the results of the random effects models are tested against a fixed effects model that does not assume $E\left(X^{\prime} \varphi\right)=\iota_{k} 0$.

Because Model 4.4 has a likelihood insignificantly different than that of Model 4.5 and has an additional degree of freedom, it is chosen for comparison in Table IV.2 with a fixed effect model in which all district effects are unconstrained. The $\beta$ parameter estimates in the two estimation schemes are almost identical. A Hausman test statistic, jointly testing the equality of the seven parameter estimates, is 0.32 ; the probability of correctly rejecting the null hypothesis of equality between the two sets of estimates is less than 0.005 . In this example, then, the use of a spatial random effects estimator is not inappropriate.

\section{CONCLUSION}

When district specific effects are uncorrelated with right side variables, there are clear benefits to spatial modeling. This paper has discussed the gains in information and efficiency that are achieved by spatial random effects modeling. 
The spatial modeling approach is relevant for a wide range of issues. In public finance, for example, spatial modeling can be used to suggest the extent to which states or nations look to others in determining the appropriate composition of taxes or tariffs, levels of expenditure, and public good provision. Research on the effects of networking within urban areas may find spatial techniques useful in identifying externalities associated with unemployment or poverty in inner cities. The extent to which changes in firm behavior are matched by competitors can also be studied using spatial techniques, where data can be used to determine both the identity of competitors $(W)$, and the extent to which correlated behavior is the result of intentional copycatting $(\phi)$, or simply the result of common shocks $(\tau)$.

Department of Economics, Harvard University, Cambridge, MA 02138, U.S.A.

Manuscript received March, 1988; final revision received May, 1990.

\section{APPENDIX}

\section{The Moran $M I$ Statistic}

Under the null hypothesis that errors are identically and independently distributed normal variates, the expected value of the $M I$ statistic is

$$
E(M I)=\frac{-d \operatorname{tr}\left[\left(X^{\prime} X\right)^{-1} X^{\prime} \Omega X\right]}{2 J(d-k)}
$$

where $\Omega$ is the $(d \times d)$ matrix of joins $\left\{\omega_{i j}\right\} ; d$ is the number of districts; $J$ is the number of joins in the system; and $k$ is the number of parameter estimates. Also under the null hypothesis, the variance of the $M I$ statistic is

$$
\begin{aligned}
& \operatorname{Var}(M I) \\
& =\frac{d^{2}\left\{4 J+2 \operatorname{tr}\left(\left[\left(X^{\prime} X\right)^{-1} X^{\prime} \Omega X\right]^{2}\right)-\operatorname{tr}\left\{\left[\left(X^{\prime} X\right)^{-1} X^{\prime}\left(\Omega+\Omega^{\prime}\right)^{2} X\right]\right\}\right.}{\left.-2\left(\operatorname{tr}\left[\left(X^{\prime} X\right)^{-1} X^{\prime} \Omega X\right]\right)^{2} /(d-k)\right\}} \\
& (2 J)^{2}(d-k)(d-k+2)
\end{aligned}
$$

See Cliff and Ord (1981) for discussion.

\section{REFERENCES}

Alessie, R., ANd A. KaPteyn (1985): "Habit Formation and Interdependent Preferences in the Almost Ideal Demand System," mimeo, Tilburg University, The Netherlands.

Anselin, L. (1988): Spatial Econometrics: Methods and Models. Dordrecht: Kluwer Academic Publishers.

Barker, R., and R. W. Herdt, with B. Rose (1985): The Rice Economy of Asia. Washington D.C.: Resources for the Future.

Case, A. C., J. R. Hines, JR., And H. S. Rosen (1989): "Copycatting: Fiscal Policies of States and Their Neighbors," NBER Working Paper No. 3032. 
Cliff, A. D., AND J. K. ORd (1981): Spatial Processes. London: Pion.

$\rightarrow$ Deaton, A. S. (1987): "Estimation of Own and Cross-price Elasticities from Household Survey Data," Journal of Econometrics, 36, 7-30.

Gaertner, W. (1974): “A Dynamic Model of Interdependent Consumer Behavior,” Zeitschrift fur Nationalokonomie, 70, 312-326.

Griffith, D. A. (1988): Advanced Spatial Statistics. Dordrecht: Kluwer Academic Publishers.

Moran, P. A. P. (1950): "Notes on Continuous Stochastic Processes," Biometrika, 37, 17-23.

Oberhofer, W., AND J. KMENTA (1974): "A General Procedure for Obtaining Maximum Likelihood

Estimates in Generalized Regression Models," Econometrica, 42, 579-590.

Pollak, R. A. (1976): "Interdependent Preferences," American Economic Review, 78, 745-763. 